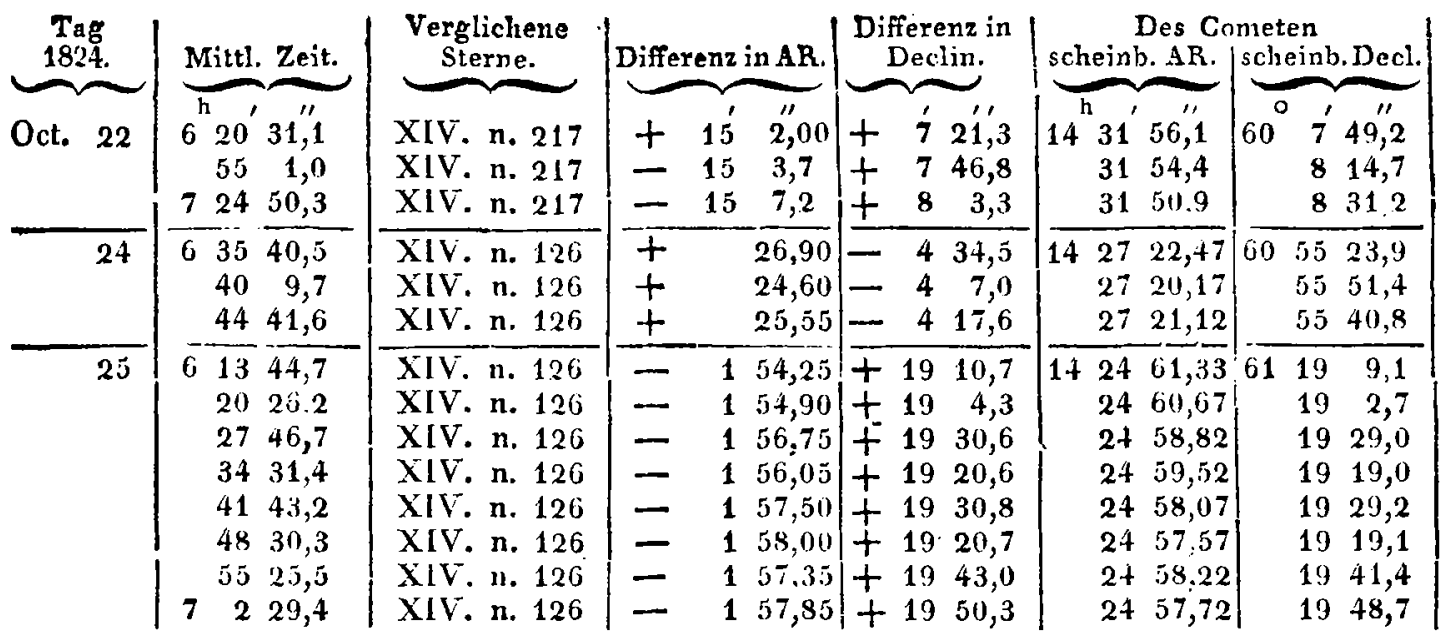

Der Comel war wegen eingetretencm Nobel so chwach, daf's derselbe nach der 3 . theobachtung nicht meler zu erliennen war.

Die Resul:ate der Beobachtungen bis zum 11ten September stehen schon Nr.6j p.283, ich habe aber mit Vergnilgen die mir hier von Herrn Littrow mitgetheilten Originalbeobachtungen wieder abgedruckt.

\title{
Ueber die Länge von Nyköping in Schweden.
}

Nach dem IV. Supplementbande der Berl. Astron. Jahrb. S. 91 rind 94 haben die Herren Schulten u. Hallströn durch Spiegelsextanlen u. Chronometer die geograph. Lage von Nyköping auf folgende Art bestimmt. Im J. 1801 fand jener die Breite des Orts $58^{\circ} 45^{\prime} 15^{\prime \prime}$, die Länge $34^{\circ} 37^{\prime} 4^{\prime \prime}$, und dieser in J. 1804 die Breite $16^{\circ} 45^{\prime} 33^{\prime \prime}$, die Länge $34^{\circ} 41^{\prime} 32^{\prime \prime}$. Im Mittel aus dieser gedoppelten Bestimmung mülste vorläufig die Breite von $\mathrm{Ny}$ köping zu $59^{\circ} 45^{\prime \prime} 24^{\prime \prime}$ und die Länge zll $58^{\circ} 37^{\prime \prime}, 2$ in Zeit von Paris angenommen werden; inders weichen besonders die Längenbestimmungen beider Beobachter stark von einander ab. Vor 4 Jahren hat Herr Prof. Cronstrand aus Stockhulm, als er, mit einer Dreiecksmessung beschäftigt, in Nyköping anwesend war, zwei astron. Beobachtungen zum Behuf der Länge daselhst gemacht. Am 7. Sept.1820 beohachtete er das Ende der Sonnenfinsterni Is um 4 h $15^{\prime} 59^{\prime \prime}, 0 \mathrm{~m}$.Z. Daraus findet sich mit denjenigen Elementen, deren ich mich im Astr. Jahrb. 1825 bedient habe, die verbesserte Conjunctionszeit 2 h $58^{\prime} 3^{\prime \prime}, 98$ und damit, durch Vergleichung mit dem Ende zu Paris, die Länge von Nyköping $+58^{\prime} 47^{\prime \prime}, 3$. Kurz zuvor, am 29. Aug. 1820, hatte Hr. Cronstrand ebendaselbst die Bedeckung der Alcyone beobachtet. Der Stern trat ein um $9 \mathrm{~h} 48^{\prime} 38^{\prime \prime}, 2$ bis $36^{\prime \prime}, 2$ und aus um $10 \mathrm{~h} 27^{\prime} 4 j^{\prime \prime}, 8 \mathrm{~m}$. $\mathrm{Z}$. Indem ich zur Berechnung dieser Bedeckung für den $O_{1} t$ des Monds und der Plejaden und für die Breitenverbesserung dieselben Elemente anwandte, mit denen Herr Rosenberger (Astron. Nachr. Nr.50.) die Rechunng geführt hat, erhielt ich aus dem Austritte die Conjunction 10h $36^{\prime} 41^{\prime \prime}, 15$, der Austritt zu Bremen gab die Conjunction 10h 3' 53",61 und daraiss folgt, wenn die Länge von Bremen zu $25^{\prime} 54^{\prime \prime}$ angenommen wird, die Länge von Nyköping 58' 41",5. Das Mittel aus diesen beiden neueren Beobachtungen gäbe die Länge von $58^{\prime} 44^{\prime \prime}, 4$. Inde「s hält Hr. Prof. Cronstrand die Sternbedeckung für genaner, als die Sonnenfinsternifs, wiewohl er über die Zeit beider Beobachtungen wegen des nicht ganz regelmälsigen Gangs seines Chronometers nicht vollkommen gewifs ist. Die Breite von Nyköping setzt übrigens $\mathrm{Hr}$ Cronstrand, mit obigen Angaben übereinstimmend, $58^{\circ} 45^{\prime} 24^{\prime \prime}$ und die Länge nimmt er an zu 58' 46"', letztere, wie Hr II cullströn sie fand.

Stuttgart 1824. Oct, 26.
W $u r m$

Verzeichnifs derjenigen Instrumente, welche in dem mathematisch-mechanischen Institute (von Reichend bac/ und Ertel in München) um nachstchende Preise verfertigt werden.

Alle Objective, Okulare und Libellen sind aus dem optischen Institute (Utzschneider und Fraunhofer in München.

Passagen-Instrument Preis fl. 2200

mit achromatischem Fernrohre, dessen Objectiv 6 Fuls Brennweite, und 4 Zoll 4 Linien Oeffnung hat, mit einem Niveau zum Anhängen an die Achse, 4 astronomischen Okularen, nnd einem Sonnenglas. Die Beleuchtung geschieht durch die HorizontalAchse.

Passagen-Instrument Preis fl. 900 mit achromatischem Ferurohre von 3 Fu「s 6 Zoll Brennweite, und 2 Zoll 10 Linien Oeffnung, nebst Nivean zum Anhängen an die Achse, 3 astronomischen Okularen und einem Sonnenglas.

\section{Meridian-Kreis Preis fl. 4200}

von 3 Fuls im Durchmesser, mit silbernem Limbus, und vermittelst der 4 Verniers vnn 2 zu 2 Sekunden getheist. Das achromatische Fernrolur hat 5 Fuls Brennweite, 4 Zoll 3 Linien Oeffnung, 4 astronomische Okulare und 1 Somengias. Der gamze Meridianliseis ist wie ein Passagen-Instriment zur 
Rectification, und Untersuchung des Collimations-Fehlers zum Umhärgen eingerichtet, in allen seinen Theilen vollständig balancirt, und hat 2 grolse Niveall, eines zum Anhängen an die Horizontal-Achse, das andere zur Versicherung des festen Standes der Verniers. Die Fädenbeleuchtung geschieht durch die Achse, und das Okular ist zum Verschieben eingerichtet.

\section{Meridian-Kreis Preis fl. $\mathbf{1 8 0 0}$}

ron 20 Pariser Zoll im Durchmesser, mit silbernem Limbus, und vermittelst der 4 Verniers von 4 zu 4 Sekunden getheilt. Das achromatische Fernrohr hat 42 Zoll Brennweite, und $34 \mathrm{Li}$ nien Oeffuung, 3 astronomische Okulare, und 1 Sonnenglas. Der ganze Meridiankreis ist, wie ein Passagen-Instrument zur Rectifikation, und Untersuchung des Collimatians-Fehlers zum Umhäugen eingerichtet, in allen seinen Theilen vollständig baJancirt, und hat 2 Niveau, eines zum Anhängen an die Horizontal-Achse, das andere zur Versicherung des festen Standes der Verniers. Die Fädenbeleuchtung geschjeht durch die Achse, und das Okular ist zum Verschieben eingerichtet.

\section{Repeticions-Kreis Preis fl. 4100}

von 3 Fufs im Durchmesser mit stehender Säule, und einem Azimuthalkreis ron 2 Furs im Durchmesser, beide Kreise mit silbernem Limbus, ersterer giebt durch 4 Nonien 2, und letzterer durch 2 Nonien 4 Secunden. Das achromatische Objektiv des Fermohrs hat 4 Fufs Brennweite, und 3 Zoll 1 Linie Oeffrung. Das prismatische Okular hat 4 Einsätze mit cinem Somuenglas. Die Fädenbeleuchtung geschieht durch die Achse des Fernrohres. An der Vertikal-Achse ist ein grolses Niveal befindlich, und zur Versicherung des unverriickten Standes des Kreises beim Umdrehen der Alhidade wird ein Fühlhebel, welcher statt eines beweglichen Niveau's dient, angebracht. Eir 2tes Niveau dient zum Anhängen an die Horizontal-Achse.

Transportabler astron. Mulliplikations-Kreis Preis $\mathbf{f l} 1600$ von $18 \mathrm{Zoll}$ im Durchmesser mit einem Azimuthalkreise von $8 \mathrm{Zoll}$ im Durchmesser, beide Kreise mit sjlbernem Limbus; ersterer mit 4 Verniers von 4 zu 4 Sekunden, letzterer durch 1 Vernier von Minute zu Minute getheilt. Das achromatische Objektiv hat 2 Furs Brennweite, 1 Zoll 10 Linien Oeffnung. Das prismatische Okular hat einen Einsatz nebst einem Sonnenglas. Die Fädenbeleuchtung geschicht durch die Achse des Fernrohres. An der Vertikal-Achse ist ein grofses Niveau befindlich, und zur Versicherung des miverriickten Standes des Kreises beim Umdrehen der Alhidade ist ein bewegliches Niveau angebracht. Ein 3tes Niveau dient zum Ambängen an die Horizontal-Achse.

Transportables Universal-Instrument Preis fl. 1600 mit Höhenkreis von 10 Zollen, Aufsuchungskreis von 10 Zollen, Azimuthalkreis von $5 \mathrm{Zoll}$, und Horizontalkreis von $13 \mathrm{Zoll}$ im Durchmesser, erster mit 4 Verniers von 4 zu 4 Sekunden, zweiter mit 1 Vernier von 10 zu 10 Sekunden, dritter mit 1 Vernier von Minnte zu Minute, und vierter mit 4 Verniers von $4 \mathrm{zu}$ 4 Sekunden getheitt. Das erste Fernrohr, welches mit der $\mathrm{Ho}$ rizontal-Achse, wovon der Höhenkreis zum Multipliciren mit einer beweglichen Libelle versehen ist, hat 1 Zoll und 9 Linien Oeffnung, und 18 Zoll Brennwejte. Das Perspektiv für den Horizontalkreis hat $16 \mathrm{ZoII}$ Brennweite, und 15 Linien Oeffnung.

Multiplikations-Theodolit Preis fl. 800

ron 12 Zollen im Durchmesser mit 4 Verniers auf silbernem Linıbus von 4 zu 4 Sekunden getheilt, mit einem Höhenkreis ron 6Zoll im Durchmesser, von Minute zu Minute auf silbernem
Limbns getheilt, 2wei achromatischen Femröhren von $16 \mathrm{Znll}$ Brennweite, 15 Linien Oeffuung, 1 astronomischen Okular, 1 Sonnenglas, und Niveau zum Aufstecken auf die Horizontalachse.

\section{Multiplications-7heodolit Preis fH. 500}

von 8 Zoll im Durchmesser mit 4 Verniers von 10 zn 10 Sekunden auf silbernem Limbus getheilt, mit einem Höhenkreise von $5 \frac{1}{2} \mathrm{Z}$ oll durch den Nonius von Minute zu Minute auf silbernem Limbus getheilt, 2 achromatischen Fernröhren von $10 \mathrm{Zoll}$ Brennweite, 12Linien Oeffunng, einem astronmischen Oculare, 1 Sonnenglas, und Niveau zum Aufstecken auf die Horizontal-Achse.

\section{Kleiner Theodolit Preis fl. 200}

von 6 Zoll im Durchmesser mit Höhen-Gradbogen auf silbernem Limbus von Minute zu Minnte getheilt, 2 achromatischen Fernröhren von 8 Zoll Länge, und einem Niveau. Das ganze Instrument befindet sich auf einem Gestelle von 3 hölzernen Fürsen.

Astronomischer Multiplications-7heodolit Preis $\mathbf{1 . 6 0 0}$ von $S$ Zoll im Durchmesser mit 4 Verniers von 10 zu 10 Sekunden anf silberuem Limbus getheilt, mit einem Azimuthalkreis von 4 Zoll Durchmesser durch den Nonius von Minute zu Minute auf silbernem Limbus getheilt, achromatischen Fernröhren von 12 Zoll Brennweite, 12 Linien Oeffulug, einem astronomischen und prismatischen Okulare, 1 Sonmenglas, 2 Niveau's, das eine zum Aufstecken auf die Horizontal-Achse, das andere zum Aufstecken auf die senkrechte Achse.

\section{Aequatoreal-Instrument Preis fi. 4000}

dessen Achse 3 Furs 9 Zoll lang ist, mit einem Stunden-und Declinations-Kreis, jeder von $2 \mathrm{FuIs}$ und $3 \mathrm{Zoll}$ im Durchmesser mit silbernem Limbus. Ersterer giebt durch 2 Vernier's, 4 Sekunden in Zeit, letzterer 4 Sek. in Raum. Das achromatische Fernrohr hat 3 Fufs 6 Zoll Brenuwejte, 3 Zoll 2 Linien Oeffnmm. 3 astronomische Okulare, nebst einem prismatischen Okular, 1 Sonnenglas, 3 Niveau's, und einem Kreis - und Filar-Mikrometer; letzteres zum Repetiren. Die Fädenbelenchtung geschieht durch die Achse des Ferntohres.

NB. Alle Bestellungen werden direct bei dem Unterzeichneten in München, Maxvorstadt Karls - u. Louisenstrafse Nr.203 gemacht.

\section{T. $E ; \quad r \in l$,}

Inhaber des obgenannten mathemat.-mechan. Instituts.

NB. Distanzenmesser ohne Latte . . . . . fl. 140.

Ein Nivellir-Instrument mit einem Horizontal-Kreis, einer

Libelle zum Umhängen, der Nonius giebt eine Minute.

Das Ganze rulht auf 3 hölzernen Fiifsen $\cdot \dot{ } \cdot-$ Verhältnils.

Merstisch nach nevester Construction mit hölzernen Fiirsen, und Obertheil ganz von Messing.

MeIstisch wie obiger, beim Obertheil sind die Schirauben von Messing, das übrige von Holz . . . . -

Kippregel mit einfachem Fernrohr, Diopter-Gradbogen und Lincal .

Stangenzirkel mit hölzerner Stange, sanfter Bewegung und

Mikrometerschraube . . . . . $\quad-15$

Kopier-Maschine mit messing. Sänle u. eisemem Corippe :

do. do. mit hölzerner Sänle und Gerippe - - 166

Boussole mit Nadel von 4 Zoll Länge und Diopter : - 20.

do. ohne Diopter . . . . . . - 13.

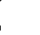

